\title{
Detection and Identification of Defects in Transparent Film
}

\author{
Bao-yuan Chen, Chao Zheng, Zhong-xiang Sun and Xiao-yang Yu \\ The higher educational key laboratory for Measuring \& Control Technology and \\ Instrumentations of Heilongjiang Province Harbin University of Science and \\ Technology, Harbin 150080,China \\ chenbaoyuan@126.com
}

\begin{abstract}
Biaxially oriented polyester film (BOPET) defect is an important factor affecting the quality of the film. In view of identification of defects in the conventional film production process, this mathod resulted in the identification of defects inaccurate. and low labor efficiency and machine vision recognition on identification of specific defect. This paper presents a LVQ neural network-based BOPET film of defects detection and identification methods. In this algorithm, the film images were processed and the outlines of the membrane defects were obtained. Through extracting the aspect ration, circularity, complexity and elongation, projection histogram central moment and so on, the characteristic values of membrane defects, which from the image of film images after image processing, and then input to the defects recognition system based on LVQ neural network that had been trained, in order to achieve the film defects identification, classification and localization. Through the study of features of the defects in BOPET and extracted some quantities as character input of the LVQ neural network, then input some characteristic values as training value into the LVQ neural network to achieve the learning and prediction purpose, and the LVQ neural network was designed. The experiments show that, the proposed method can meet the requirements analysis of air defects in transparent film.
\end{abstract}

Keywords : LVQ ; Sobel edge detection ; identification defects ; Transparent Film

\section{Introduction}

Biaxially oriented polyester film (BOPET) is a plastic packaging materials most widely used one, common thickness of $12 \mu \mathrm{m}$, with its excellent physical and mechanical properties and is widely used in composite packaging, printing, vacuum aluminum and other fields . BOPET has high mechanical strength, heat resistance, transparency, nontoxic, folding, small permeability, good electrical insulation properties ${ }^{[1][2][3]}$. In the film production process, defects thin film is an important quality factor. Commonly used detection methods are manual detection and identification and machine vision detection and identification. The traditional methods of detection and identification have manual labor intensity, low detection accuracy. In recent years, there have been automated detection of impurities in plastic film studies ${ }^{[4][5]}$, but they are based on the specific type of impurity identification, and can not distinguish between types of impurities.

In this paper, a film based on LVQ neural network defects detection and identification methods, by acquiring the film image, after image pre-processing to extract the aspect ratio, circularity, shape complexity, elongation and projection histogram central moment in ten eigenvalues and other characteristics as LVQ neural network input value, after a judge to identify the type of defects in the film, and give its location and size. 


\section{BOPET Film Acquisition and Image Processing}

The film has good smoothness, transparent, non-texture features.By artificial light is irradiated on the film, the film will be apparent defects . Image acquisition device is typically used CCD sensor, which will not only be easy to use and low cost. In the image acquisition process, various disturbances will be subject to external factors, the need for pre-processing the collected images. After a series of processing, film defects detected image and feature extraction, identify the defects type according to LVQ neural network .

Detection and identification of defects during the film generally includes image preprocessing, edge detection, identify the type, size and centroid calculation of defects ,determined as follows:

(1) Image pre-processing: image preprocessing includes gray, smooth de-noising, filter out the noise, edge information can to make it easier to extract;

(2) Image segmentation: Select thresholding method for image binarization processing, post-processing defects obvious contrast with the background;

(3) Edge detection: then by the Sobel edge detection, get the target image edge;

(4) Morphological image processing: to get a complete outline defects through the edge of the defects film defects closed, filling and other operations;

(5) The feature extraction: contour extraction target image characteristic value, the extracted feature value as the judgment LVQ neural network input.

(6) Target recognition: the defects extracted image input to the network has been trained to identify the type defects and give its location and size.

Flow chart of film defects extracted shown in Figure 1:

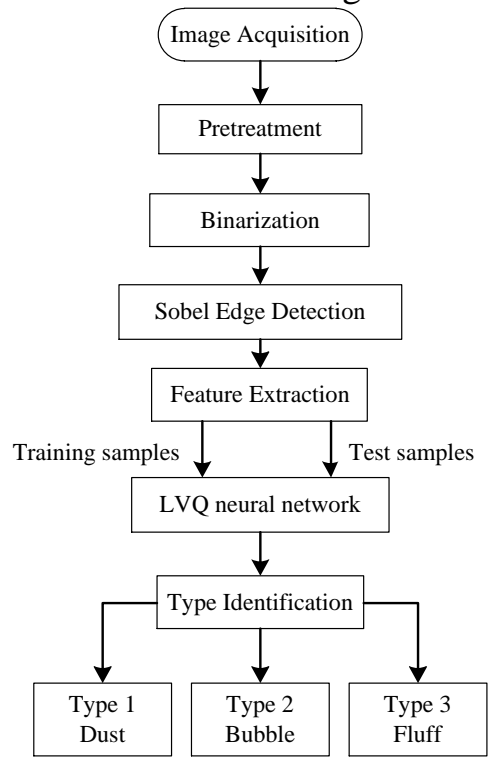

Figure 1. LVQ Neural Network Target Identification Flowchart

\section{BOPET Film Image Preprocessing}

Many types of film of defects, according to the source of defects can be broadly divided into foreign matter such as dust, lint, oil, etc., and the film itself impurities such as bubbles, gel spots and scratches. Due to the action of the gas bubbles are produced, generally into a round or oval shape, the edge of the bubble than the light, with a clear boundary ${ }^{[6]}$. Dust images are generally black dot, fluff linearly irregular and so on. This paper studies detection and identification of the bubbles, lint and dust, three defects. Defect image shown in Figure 2. 


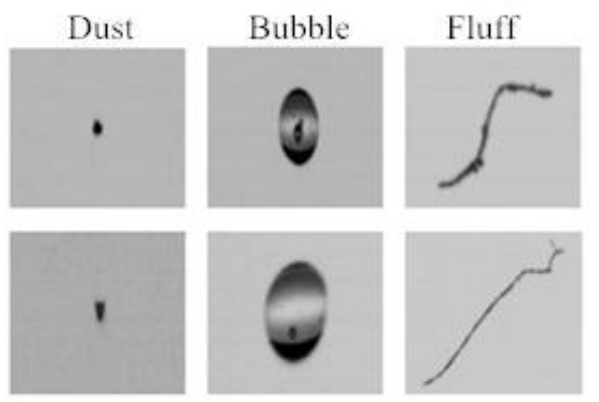

Figure 2. Film Defects

\subsection{Denoising}

In the image acquisition process, due to the impact the accuracy of the camera, lighting conditions and other factors, the captured image will be some random noise, the main purpose is to reduce the image smoothing image noise. Median filter is easy to remove outliers and background impurities share and protect the image edges while removing the noise, the image quality is greatly improved. Not only mean filter to remove interference, often blurring the edges of the image, resulting in distortion, we use both to noise interference, but also a very good edge retention detail median filter algorithm ${ }^{[7] .}$

Principle median filter is to select a first point of an odd number of window $\mathrm{W}$, the window will be scanned on the image, the pixels contained in the window according to the gray level up (or down) arranged in order, in the middle of taking gray value, instead of the gray value of the point, i.e.,

\subsection{Image Segmentation}

$$
\mathrm{g}(\mathrm{m}, \mathrm{n})=\operatorname{Media}\{\mathrm{f}(\mathrm{m}-\mathrm{k}, \mathrm{n}-1),(\mathrm{k}, 1) \in \mathrm{W}\}
$$

Thresholding is the area through the image into regions outside the internal point set point set to the regional-based. Common binarization threshold segmentation is the image gray value is greater than the pixel set threshold value $\mathrm{T}$ is white (set to 1), the gray value is less than the threshold value $\mathrm{T}$ of the pixel is set to black (set to 0 ), so that so that the original grayscale image $f(x, y)$ converted into black and white binary image $g(x, y)$. After binarization threshold processing image $\mathrm{g}(\mathrm{x}, \mathrm{y})$ shown in equation $(2)$ below:

$$
g(x, y)= \begin{cases}1 & f(x, y)>T \\ 0 & f(x, y) \leq T\end{cases}
$$

In thresholding, select the threshold value directly affects the results of image segmentation. In this article, since the film image is generally uniform gray background image and background differences more obvious defects, solid selection of global thresholding method. After several experiments, the choice of threshold $\mathrm{T}=165$. Image threshold segmentation method divided in Figure 3.

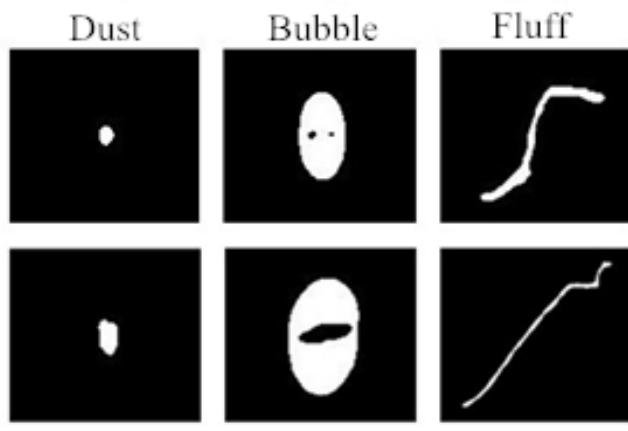

Figure 3. Threshold Image Segmentation 


\subsection{Edge Detection}

Image edges are basic characteristics. It is not sensitive to changes in gray, is a collection of those pixels in the image pixel gray rapidly changing, including the main information of the target object boundary ${ }^{[8]}$. The basic idea is to check the edge detection of the edge points in the image, and then follow an algorithm connected to the contour edge points, thereby forming the divided regions.

Sobel operator is a edge detection method based on gradient, which is weighted average of its neighbors, and weighted gray value difference between the upper and lower right of the image around each pixel neighbors domain. Its neighborhood is close to the maximum. Sobel operator template is shown in Figure 4. $G_{x}$ gradient is corresponding to pixels in the horizontal direction, which is used to detect vertical edges.And $G_{y}$ is corresponding to pixel in the vertical direction, wit is used to detect horizontal edges.

\begin{tabular}{|c|c|c|}
\hline-1 & 0 & 1 \\
\hline-2 & 0 & 2 \\
\hline-1 & 0 & 1 \\
\hline
\end{tabular}

$\left(G_{x}\right)$

\begin{tabular}{|c|c|c|}
\hline 1 & -2 & -1 \\
\hline 0 & 0 & 0 \\
\hline 1 & 2 & 1 \\
\hline
\end{tabular}

$\left(G_{y}\right)$

\section{Figure 4. Sobel Operator Template}

Make convolution operation using the above operator according to formula (a) and formula (b), and obtain the pixel value of the gradient: $|\mathrm{fx}|+|\mathrm{fy}|$. Edge points are greater than or equal to the gradient threshold value points, otherwise not, to achieve edge detection.

$$
\begin{aligned}
\Delta f_{x}(x, y) & =\{f(x+1, y-1)+2 f(x+1, y)+f(x+1, y+1)\}-\{f(x-1, y-1)+2 f(x-1, y)+f(x-1, y+1)\} \\
\Delta f_{x}(x, y) & =\{f(x-1, y+1)+2 f(x, y+1)+f(x+1, y+1)\}-\{f(x-1, y-1)+2 f(x, y)+f(x+1, y-1)\}
\end{aligned}
$$

Sobel operator simply has to pay efficiency for edge detection, wide range of applications. Due to the film impurities image to be processed by this article, changes in the target and background pixel values of adjacent pixels are intense, and the target edge points are extremely reached, target edges are easily separated from the background. So use Sobel operator for image segmentation. Film impurities edge detection results shown in Figure 5, the bubble by internal image edge detection after a cavity.

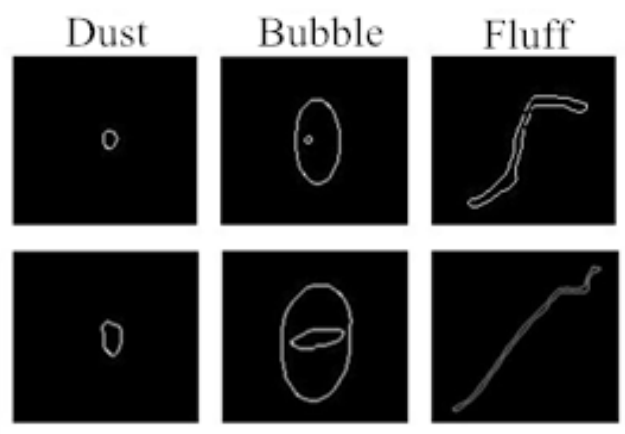

Figure 5. Edge Detection Image 


\subsection{Binary Image Morphological Processing}

The binary image is a special image processing time, only two gray levels 0 and 1 , with a small space features. Erosion and dilation are two basic operations morphological processing. Assume that A is a region to be processed, B the structural element, A to B with corrosion is defined as

$$
\mathrm{A} \oplus \mathrm{B}=\left\{\mathrm{x} \mid(\mathrm{B})_{\mathrm{x}} \subseteq \mathrm{A}\right\}
$$

$\mathrm{A}$ is expanded $\mathrm{B}$, defined as

$$
\mathrm{A} \ominus \mathrm{B}=\left\{\mathrm{x} \mid\left[(\widehat{\mathrm{B}})_{\mathrm{x}} \mathrm{nA} \subseteq \mathrm{A}\right]\right\}
$$

Filling the image area is that filling and empty in the binary image. Known set of image boundary, the filling process of the boundary area is surrounded by a point $\mathrm{P}$ in the region from the beginning to make the iteration, when $X_{k}=X_{k}-1$ iterations stop, then, the intersection set of the $\mathrm{X}_{\mathrm{k}}$ and $\mathrm{A}^{\mathrm{c}}$ contains a fill region and its side ${ }^{[9]}$.

$$
\mathrm{X}_{\mathrm{k}}=\left(\mathrm{X}_{\mathrm{k}-1} \oplus \mathrm{B}\right) \cap \mathrm{A}^{\mathrm{c}}, \mathrm{k}=1,2,3, \ldots
$$

Corrosion in the role of mathematical morphology is to eliminate the boundary points of the object, eliminating irrelevant details. In contrast with the corrosive effects, the role of expansion of is the image boundary expansion, and the cracks connected. After pretreatment and edge detection image obtained some non-closed contour, and there are some unrelated edges and isolated point inside and outside edges. Expansion and corrosion can solve the above problems ${ }^{[10]}$. After Filling empty image shown in Figure 6:
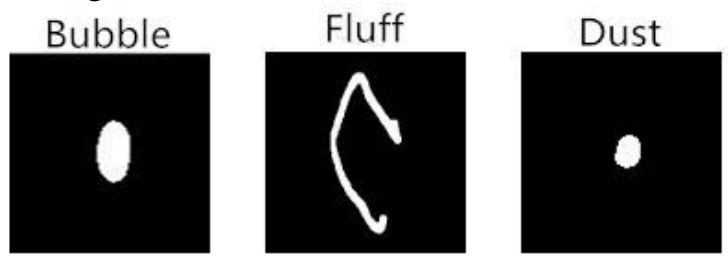

Figure 6. Fill Empty Image

\section{Defect Feature Extraction and Recognition Algorithm}

After the film image processing, various impurities in the film profile can be obtained. According to the image of the defects of the film to extracte, The characteristic values extracted LVQ neural network as input, by judgment to identify bubbles, dust, fluff and three impurities neural network, and gives the location and size of the impurities.

\subsection{Film Defect Feature Extraction and Recognition}

Feature extraction is the key step in the image of image recognition, according to the characteristics of the image identification and classification ${ }^{[11]}$. Edge of the bubble is smooth, and there is a clear boundary. Dust images are generally black dot, fluff and irregularly shaped and so linearly. So the aspect ratio, circularity, complexity, elongation characteristics are extracted as the shape feature. Projection histogram of the image reflect the distribution of the histogram. And the first three central moments obtained could describe the features of the defects. So the selection of aspect ratio (Aspect Ration), circularity (Circularity), shape complexity (Complexity), elongation (Elongation) and horizontal and vertical projection histogram central moment $\left(\mathrm{M}_{\mathrm{x} 1}, \mathrm{M}_{\mathrm{x} 2}, \mathrm{M}_{\mathrm{x} 3}, \mathrm{M}_{\mathrm{y} 1}, \mathrm{M}_{\mathrm{y} 2}\right.$, My3) 10 feature extracted ${ }^{[12]}$, as input variables LVQ neural network input. Among 


$$
\begin{aligned}
\text { Aspect Ration } & =\frac{\text { Maxlength }}{\text { Minlength }} \\
\text { Circularity } & =\frac{4 * \pi * \text { Area }^{2}}{\text { Perimeter }^{2}} \\
\text { Complexity } & =\frac{\text { Perimeter }^{2}}{\text { Area }} \\
\text { Elongation } & =\frac{\text { Maxlength }- \text { Minlength }}{\text { Maxlength }+ \text { Minlength }}
\end{aligned}
$$

The first three central moments of projection histogram are

$$
\begin{aligned}
& \mathrm{M}_{1}=\frac{1}{\mathrm{~L}} \sum_{\mathrm{i}=1}^{\mathrm{L}} \mathrm{P}(\mathrm{i}) \\
& \mathrm{M}_{2}=\sqrt{\frac{1}{\mathrm{~L}} \sum_{\mathrm{i}=1}^{\mathrm{L}}\left(\mathrm{P}(\mathrm{i})-\mathrm{M}_{1}\right)^{2}} \\
& \mathrm{M}_{3}=\sqrt[3]{\frac{1}{\mathrm{~L}} \sum_{\mathrm{i}=1}^{\mathrm{L}}\left(\mathrm{P}(\mathrm{i})-\mathrm{M}_{1}\right)^{3}}
\end{aligned}
$$

For the determination of the number of training samples is commonly used rule of thumb the way, so this experiment using 300 different samples (200 training samples and 100 test samples) experiment,which not only effectively reduce the input of LVQ networks, but also for the establishment of efficient and stable neural network to do the preparation.

The image of the BOPET after processing selects eigenvalue calculated by Matlab programming, in order to obtain eigenvalues. Part of the training sample test results will be randomly selected in a tabular form, as shown in table 1 .

Table 1. Part of the Training Sample Analysis Results

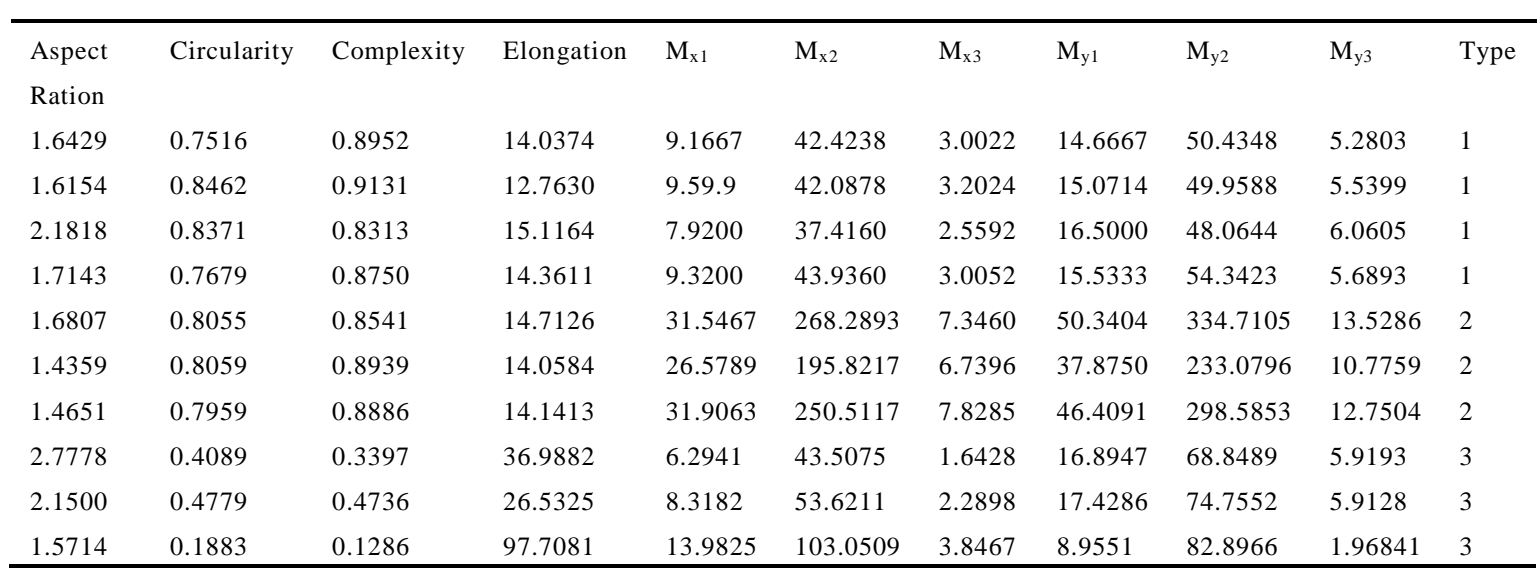

\subsection{LVQ Neural Network Structure Design}

LVQ neural network is also known as learning vector quantization LVQ (Learning Vector Quantization) neural network. It is a supervised learning neural network application integration and unsupervised learning rule. LVQ neural network develops from learning Lenovo and algorithm competition, because of its simple structure characteristics in the field of pattern recognition and optimization has been widely used[13,14].

LVQ neural network is a two-tier network architecture, the first layer is a competitive layer, the second layer is a linear layer. Competitive layer is used for classifying the input vector; the linear layer is to convert the classified information to the user's expectations category. Competitive layer and linear layers have one 
neuron for each category. Assuming that the competitive layer learns to get the $S^{1}$ subclasses, and the subclasses are combination with the competitive layer $S^{2}$ expectations category. LVQ neural structures is shown in Figure 7.

LVQ learning algorithm is as follows:

(1) The input and output samples:

$$
\left\{\mathrm{p}_{1}, \mathrm{t}_{1}\right\},\left\{\mathrm{p}_{2}, \mathrm{t}_{2}\right\}, \ldots,\left\{\mathrm{p}_{200}, \mathrm{t}_{200}\right\}
$$

There are 10 elements of the input vector, the input feature vector consisting of 10 feature vectors. Desired output vector is only one element is 1 , and the rest are 0.1 represents the expectations associated with the input category. 200 film defect image samples divided into three categories ( 1 dust, 2 bubble, 3 fluff). Each sample is divided into one of three categories. Initialize the weight matrix IW 1,1 , set the initial learning rate $\alpha=\alpha(0)$.

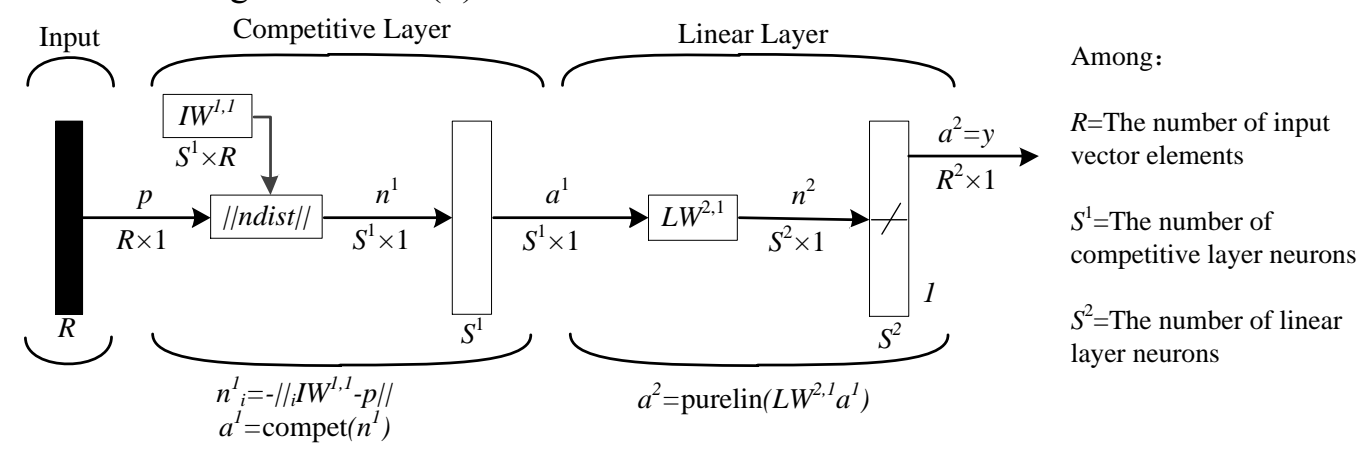

Figure 7. LVQ Neural Network Model

(2) The distance of the input vector $p$ and the input weight matrix IW1,1apply ndist () function to calculate to find the minimum distance from the input vector. The hidden layer is the competition layer. Assuming the $\mathrm{i}$-th element of $\mathrm{n} 1$ wins the completion, the competitive output is a1,which the $\mathrm{i}$-th element is 1 , and other elements are 0 .

(3) Adjust the weight matrix $\mathrm{IW}^{1,1}$, i-th row 1, if the classification is correct, it moves to the input vector $\mathrm{p}$; if the classification is incorrect, the input vector in the opposite direction to the movement. o if p classified correctly,

$$
\mathrm{a}_{\mathrm{k}^{*}}^{2}=\mathrm{t}_{\mathrm{k}}=1
$$

The weight matrix can be calculated IW1,1, the new value of the row $\mathrm{i}^{*}$ :

$$
{ }_{i} * \operatorname{IW}^{1,1}(q)={ }_{i} * W^{1,1}(q-1)+\alpha\left(p(q){ }_{-} * W^{1,1}(q-1)\right)
$$

If the classification is incorrect:

$$
a_{k^{*}}^{2}=1 \nRightarrow \mathrm{t}_{\mathrm{k}^{*}}=0
$$

The weight matrix can be calculated $\mathrm{IW}^{1,1}$, the new value of the row $\mathrm{i}^{*}$ :

$$
\mathrm{i}^{*} \mathrm{IW}^{1,1}(\mathrm{q})=\mathrm{i}_{\mathrm{i} * \mathrm{IW}} \mathrm{IW}^{1,1}(\mathrm{q}-1)-\alpha\left(\mathrm{p}(\mathrm{q}){ }_{\mathrm{i} *} * \mathrm{IW}^{1,1}(\mathrm{q}-1)\right)
$$

(4) Also select a training sample of the input vector to the LVQ network, returning to step (2) until all the vectors are available again reached.

(5) Reducing the learning rate $\alpha$, and the test stop conditions are met, if the train is stopped, otherwise return to step (2) ${ }^{[15]}$. 


\section{Results}

Experiments collected 200 different training samples to carry out under LVQ neural network training, and the final selection of the number of neurons in competitive layer is 15 , learning rate of 0.01 LVQ neural network mode as a network BOPET film impurity detection and identification system for testing. The 100 samples 100 collected from different conditions are as a test sample of the selected network, Verified by testing the trained LVQ network. Part of the training sample test results will be randomly selected in a tabular form, as shown in Table 2 .

Tabel 2. Part of the Testing Sample Analysis Results

\begin{tabular}{|c|c|c|c|c|c|c|c|c|c|c|c|}
\hline Aspect & Circularity & Complexity & Elongation & $\mathrm{M}_{\mathrm{x} 1}$ & $\mathrm{M}_{\mathrm{x} 2}$ & $\mathrm{M}_{\mathrm{x} 3}$ & $\mathrm{M}_{\mathrm{y} 1}$ & $\mathrm{M}_{\mathrm{y} 2}$ & $\mathrm{M}_{\mathrm{y} 3}$ & Type & Results \\
\hline \multicolumn{12}{|l|}{ Ration } \\
\hline 1.4615 & 0.7571 & 0.9168 & 13.7066 & 8.4500 & 35.0056 & 2.8837 & 12.0714 & 41.4064 & 4.5916 & 1 & 1 \\
\hline 1.7692 & 0.7492 & 0.8703 & 14.4384 & 8.5000 & 39.4980 & 2.7951 & 14.5714 & 48.4888 & 5.3769 & 1 & 1 \\
\hline 1.4545 & 0.7500 & 0.8983 & 13.9884 & 6.9414 & 25.9323 & 2.4492 & 9.8333 & 30.3590 & 3.8280 & 1 & 1 \\
\hline 1.9000 & 0.4562 & 0.8761 & 14.3436 & 6.6500 & 27.5819 & 2.2721 & 12.0909 & 33.4403 & 3.8280 & 1 & 1 \\
\hline 1.9778 & 0.8057 & 0.7863 & 15.9815 & 31.6444 & 296.0567 & 6.9630 & 61.9130 & 409.9015 & 16.8674 & 2 & 2 \\
\hline 1.8333 & 0.8061 & 0.8291 & 15.1578 & 22.3571 & 163.3825 & 5.7067 & 40.3871 & 215.6364 & 12.3288 & 2 & 2 \\
\hline 1.6182 & 0.8090 & 0.8337 & 15.0724 & 39.1889 & 366.8043 & 8.6278 & 62.9821 & 460.3599 & 16.0796 & 2 & 2 \\
\hline 1.0400 & 0.2008 & 0.1903 & 66.0489 & 8.6078 & 59.7068 & 2.2545 & 8.2830 & 59.0262 & 2.1585 & 3 & 3 \\
\hline 1.0811 & 0.1581 & 0.1682 & 74.7160 & 11.0267 & 92.8348 & 2.5419 & 10.2099 & .5322 & 2.3249 & 3 & 3 \\
\hline 1.2000 & 0.2067 & 0.2561 & 49.0622 & 8.9344 & 67.7399 & 2.2033 & 10.6863 & 74.3988 & 2.8093 & 3 & 3 \\
\hline
\end{tabular}

Through the analysis of the calculation of all test samples, its recognition accuracy was $98.5 \%$. Figure 3 shows the final impurity detection and type recognition results in Figure 2.

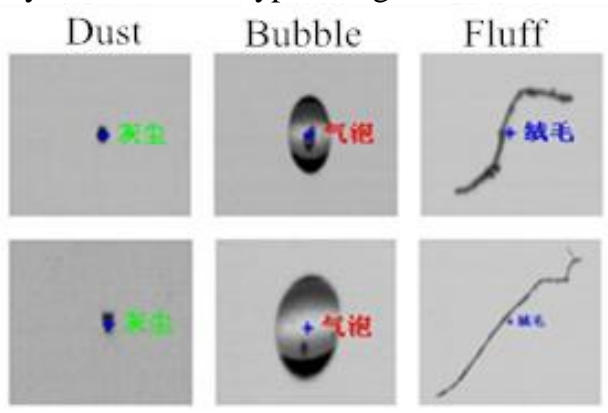

\section{Figure 8. The Result of the Identification of the type}

The location and size of defects in Figure 2 are shown in Table 3.

Table 3. Results in Figure 2

\begin{tabular}{llll}
\hline No. & Centroid & Area/pix & Type \\
1 & $(100.8900,54.8687)$ & 99 & 1 \\
2 & $(102.1410,55.8932)$ & 234 & 1 \\
3 & $(101.4737,54.7368)$ & 1330 & 2 \\
4 & $(101.2174,82.8239)$ & 2742 & 2 \\
5 & $(99.4752,78.4725)$ & 726 & 3 \\
6 & $(153.6645,103.5061)$ & 1225 & 3 \\
\hline
\end{tabular}

\section{Conclusion}

This article is compiled in Matlab R2012a environment, and selects 300 different samples (300 training samples and 100 test samples). The LVQ neural network is trained by training samples, and the competitive layer of neural network is 15 ,the learning rate is 0.01 . Through analysis all the 
test results, obtained the correct rate of identify is $98.5 \%$ and the whole detection and recognition time only $0.98 \mathrm{~s}$. The experimental data shows that the algorithm can realize the identification and location of defects in the BOPET film, and it is high precision and speed, which meet the real requirements at processing. And if the image pretreatment process is optimized, it has a higher precision and faster speed. So if this algorithm will be used in line testing equipment BOPET film is feasible. This method can also be used to detect defects in translucent material.

\section{Acknowledgment}

Instruments and equipment research project in 2014 of Harbin University of Science and Technology,No. 2012XZA04014.

This research was funded by the Scientific and Technological Project of Education Dep artment of Heilongjiang Province with Grant Number (12531113).

\section{References}

[1] F. Shuming, "Performance and modification of PET film", Polyester Industry, (2009).

[2] S. Hashemi and Y. Xu, "Thermal effects on fracture of biaxial-oriented poly (ethylene terephthalate) (BOPET) film", Journal of Materials Science, (2007).

[3] J. Sun, H.-y. Tian and Y.-p. Bai, "Graft polymerization of acrylic acid and acrylamide onto BOPET corona films", Journal of Wuhan University of Technology-Mater. Sci. Ed, (2004).

[4] L. Yan, "Key technology research of polymer film defects detection based on CCD scanning", Harbin University of Science and Technology, (2011).

[5] F. Xiangdang, "Research of Plastic Film Surface Defects Detection and Recognition", Industrial Control Computer, (2011).

[6] T. Yan, "Defect causes of dry lamination film used PET film", Polyester Industry, (2011).

[7] Z. Gao-chang, Z. Lei and W. Feng-bo, "Application of improved median filtering algorithm to image denoising”, Journal of Applied Optics, (2011).

[8] C. Han1 and L. Xingjun2, "Wheat Panicle Image Segmentation Based on Sobel Operator-edge Detection", Jornal of Agriclultural Mechanization Research, (2013).

[9] L. Li, S. Fu, T. Fang and L. A-juan, "Matlab-based extraction of interested image regions", Mordern Electronics Technique, (2013).

[10] G. Hongxia, C. Fuguo and W. Yanying, "Extraction Method for the Bubbles of Arbitrary Shape in the Components Based on X-Ray Detection", Semiconductor Inspection \& Testing Technologies, (2012).

[11] Z. Junhai, "Research on the Image Feature Extraction", Journal of Hebei University(Natural Science Deition), (2009).

[12] W. Xiaobin, K. Yaohong and L. Taijun, "Image retrieval algorithm based on image projection", Journal of Tsinghua University(Science And Technology), (2005).

[13] M. Biehl, "Admire LVQ_-Adaptive Distance Measures in Relevance Learning Vector Quantization”, KI - Künstliche Intelligenz, (2012).

[14]K. Sano, S. Momose and H. Takizawa, "Efficient parallel processing of competitive learning algorithms", Parallel Computing, (2004).

[15] Z. Mingxia, "Classification Method of Micro-Calcifications Based on LVQ Neural Networks", Computer Era, (2011). 
International Journal of Future Generation Communication and Networking Vol. 8, No. 4 (2015) 